\title{
miR-486-5p Inhibits Inflammatory Response, Matrix Degradation and Apoptosis of Nucleus Pulposus Cells through Directly Targeting FOXO1 in Intervertebral Disc Degeneration
}

\author{
Xingyu Chai ${ }^{a, b}$ Haipeng Sic Jiang Song ${ }^{b}$ Yanxue Chong ${ }^{b} \quad J^{\prime}$ Wang $^{d}$ \\ Gang Zhao
}

aDepartment of Traumatology, Shandong Provincial Qianfoshan Hospital, Shandong University, Jinan, China, 'Department of Spine Surgery, Tengzhou Central People's Hospital, Tengzhou, China, 'Department of Spine Surgery, Qilu Hospital of Shandong University, Jinan, China, dDepartment of Nursing, Tengzhou Central People's Hospital, Tengzhou, China

\section{Key Words}

$\mathrm{miR}-486-5 p \cdot F O X O 1 \cdot I D D \cdot N P$

\begin{abstract}
Background/Aims: microRNA-486-5p (miR-486-5p) and forkhead box protein O1 (FOXO1) play an important role in the development of intervertebral disc degeneration (IDD). However, their molecular mechanisms in IDD remain unknown. Methods: qRT-PCR assay was used to identify miR-486-5p expression in nucleus pulposus (NP) cells. In-vitro transfection, CCK-8, flow cytometry and luciferase reporter assay were used to validate the role and relationship of miR-486-5p and FOXO1 in lipopolysaccharides (LPS)-stimulated NP cells. qRT-PCR and Western blot were used to measure the expression levels of inflammatory cytokines, matrix degrading enzymes, and extracellular matrix (ECM)-related genes. Results: miR-486-5p expression was significantly down-regulated, while FOXO1 expression was up-regulated in LPS-treated NP cells $(P<0.001)$. miR-486-5p over-expression repressed LPS-induced expressions of inflammatory cytokines (IL-1 $\beta, I L-6$ and TNF- $\alpha$ ) and matrix degrading enzymes (MMP-3, MMP-13, ADAMTS-4 and ADAMTS-5), and promoted the expressions of LPSinhibited ECM-related genes (Aggrecan and Collagen II) (all $\mathrm{P}<0.001$ ). In addition, miR-486$5 p$ over-expression protected NP cells against LPS-induced apoptosis. However, inhibition of miR-486-5p led to the opposite effects. Mechanically, FOXO1 was a direct target gene of miR486-5p. Over-expressed FOXO1 aggravated LPS-induced injury, and antagonized protection effects of miR-486-5p. Conclusion: miR-486-5p can inhibit inflammatory response, ECM

X. Chai and H. Si contributed equally to this work.

\begin{tabular}{ll}
\hline Gang Zhao & Department of Traumatology, Shandong Provincial Qianfoshan Hospital \\
& Shandong University, No.16766, Jingshi Road, Jinan 250014, Shandong (China) \\
& E-Mail zhaogangsdqfs@163.com
\end{tabular}
\end{abstract}




\section{Cellular Physiology Cell Physiol Biochem 2019;52:109-118 \\ \begin{tabular}{ll|l} 
and Biochemistry & $\begin{array}{l}\text { DOl: 10.33594/000000008 } \\
\text { Published online: } 18 \text { February } 2019\end{array}$ & $\begin{array}{l}\text { C 2019 The Author(s). Published by } \\
\text { Cell Physiol Biochem Press GmbH\&Co. KG }\end{array}$ \\
\cline { 2 - 3 }
\end{tabular} \\ Chai et al.: miR-486-5p Regulates FOXO1 in IDD}

degradation and apoptosis in NP cells by directly targeting FOXO1, which may contribute to the biological therapy of IDD.

(C) 2019 The Author(s). Published by Cell Physiol Biochem Press GmbH\&Co. KG

\section{Introduction}

Intervertebral disc degeneration is one of the causes of lower back pain, which leads to disability and social burden [1-3]. Recently, therapies for IDD primarily aim to relieve pain symptoms, which offer only temporary benefits rather than a permanent cure [3]. The pathogenesis of IDD has been ascribed to some factors, including NP cell apoptosis, ECM loss and dysfunction of miRNAs [4-6]. Therefore, it is essential to elucidate the mechanisms underlying miRNAs-regulated IDD to develop novel and effective drugs.

MiRNAs are a kind of non-coding endogenous small RNAs with the length of $19 \sim 23 \mathrm{bp}$, and involved in regulating the expressions of complementary mRNA targets [7]. MiRNAs are not only involved in cell proliferation, apoptosis and differentiation [8], but also responsible for tissue degeneration and regeneration $[9,10]$. Previous studies suggested up-regulated miR-27a targeted PI3K expression to initiate apoptosis of NP cells [11]. Liu et al. also found the over-expression of miR-21 could inhibit phosphatase and tensin homolog gene expression to promote NP cell proliferation [12]. Notably, Ji ML et al. found the level of miR486-5p was significantly lower in IDD samples compared with controls [13]. However, the molecular mechanisms of miR-486-5p in IDD have not been elucidated till now.

In this study, we hypothesized the miR-486-5p might participate in the development of IDD. Firstly, we identified the expressions of miR-486-5p and FOXO1 in parental and LPS-treated NP cells using qRT-PCR and Western blot. Furthermore, we systematically validated the role of miR-486-5p and FOXO1 in in-vitro LPS-induced NP cells using in-vitro transfection, CCK-8, luciferase reporter assay, etc. This study will lay a new foundation for biological therapy of IDD.

\section{Materials and Methods}

\section{Cell culture}

NP cells were isolated as previously described [11]. After isolation, NP cells were resuspended in DMEM containing $10 \%$ FBS (GIBCO, NY, USA), $100 \mathrm{mg} / \mathrm{ml}$ streptomycin, $100 \mathrm{U} / \mathrm{ml}$ penicillin, and $1 \%$ L-glutamine, and then incubated at $37{ }^{\circ} \mathrm{C}$ in a humidified atmosphere with $95 \%(\mathrm{v} / \mathrm{v})$ air and $5 \%(\mathrm{v} / \mathrm{v})$ $\mathrm{CO}_{2}$. The normal human embryonic kidney cell line HEK293 was obtained from the Shanghai Cell Collection (Shanghai, China). HEK293 cells were cultured with Dulbecco's modified Eagle medium containing 10\% fetal bovine serum and $4 \mathrm{mM}$ glutamine in an incubator with $37^{\circ} \mathrm{C}$ and $5 \% \mathrm{CO}_{2}$.

\section{Transfection}

The agomir-486-5p, agomir-486-5p control, antagomir-486-5p, and antagomir-486-5p control were designed and synthesized by RiboBio (Guangzhou, China) and transfected using Lipofectamine 2000 (Invitrogen, Thermo Fisher Scientific, Inc.) into NP cells according to the manufacturer's instructions. After $24 \mathrm{~h}$ of transfection, cells were cultured in serum-free medium for $12 \mathrm{~h}$, and then treated with LPS (10, $100,500,1000 \mathrm{ng} / \mathrm{ml}$ ) for $24 \mathrm{~h}$ with normal saline as control (LPS, $0 \mathrm{ng} / \mathrm{ml}$ ). They were then harvested for subsequent experiments. For FOX01 overexpression, pcDNA-FOXO1, and pcDNA-control were obtained from RiboBio (Guangzhou, China). These vectors were transfected to cells following manufacturer's information.

\section{CCK-8 assay}

Cell proliferation was assessed by CCK- 8 assay (Dojindo Laboratories, Japan). Cells $\left(1 \times 10^{4}\right)$ were seeded into 96 -well plates and incubated at $37^{\circ} \mathrm{C}$ for $24 \mathrm{~h}$ before transfection. CCK-8 solution $(10 \mu \mathrm{l})$ was added to each well $48 \mathrm{~h}$ after transfection. After $2 \mathrm{~h}$ of incubation at $37^{\circ} \mathrm{C}$, the absorbance at $490 \mathrm{nM}$ was measured using Spectra Max 250 spectrophotometer (Molecular Devices, USA). 


\section{Cellular Physiology Cell Physiol Biochem 2019;52:109-118 \\ \begin{tabular}{ll|l} 
and Biochemistry & $\begin{array}{l}\text { DOl: 10.33594/000000008 } \\
\text { Published online: } 18 \text { February } 2019\end{array}$ & $\begin{array}{l}\text { C 2019 The Author(s). Published by } \\
\text { Cell Physiol Biochem Press GmbH\&Co. KG }\end{array}$ \\
\cline { 2 - 3 }
\end{tabular} \\ Chai et al.: miR-486-5p Regulates FOXO1 in IDD}

\section{Cell apoptosis assay}

Cell apoptosis was measured using an Annexin V-propidium iodide (PI) apoptosis detection kit (BD Biosciences, Franklin Lakes, USA). Following different treatment, cells were incubated with fluorescein isothiocyanate (FITC)-Annexin V (5 $\mu \mathrm{l})$ for $15 \mathrm{~min}$ at room temperature in the dark, and then with PI ( $5 \mu \mathrm{l})$ for $5 \mathrm{~min}$ at $4^{\circ} \mathrm{C}$ in the dark prior to analysis by flow cytometry.

\section{Quantitative real-time PCR ( $q$ RT-PCR)}

Total RNA was extracted using Trizol reagent (Invitrogen, Carlsbad, CA, USA). Reverse transcription was performed using the First Strand cDNA Synthesis Kit (Takara, Shiga, Japan). Quantitative PCR (qPCR) was performed using an ABI Prism 7900HT Sequence Detection System (Applied Biosystems, Foster City, CA, USA) with SYBR Premix Ex Taq II (Takara, Shiga, Japan). To quantitate miR-486-5p expression, total RNA was polyadenylated and reverse transcribed using an All-in-One ${ }^{\mathrm{TM}}$ miRNA qRT-PCR Detection Kit (GeneCopoeia, Rockville, MD, USA), according to the protocol. The expression of each target gene was normalized to that of U6. Quantification was calculated using the $2-\Delta \Delta \mathrm{CT}$ method.

\section{Western blotting}

Total proteins were extracted and separated by 10\% SDS-PAGE and transferred onto a PVDF membrane (Millipore, USA). To block nonspecific binding, the membranes were incubated with $5 \%$ skim milk powder at room temperature for one hour. The membrane was then incubated with primary antibody $(1: 1000$, Abcam, USA), followed by HRP-labeled secondary antibody (Santa Cruz) and detected by chemiluminescence. An anti-GAPDH antibody (1:1000, Santa Cruz) was used as a protein loading control.

\section{Luciferase reporter assay}

The 3'-UTR of FOXO1 was found to possess a putative miR-486-5p binding site. The WT or MUT 3'-UTR segment containing the putative miR-486-5p-binding site was amplified and inserted into the pGL3 control vector (RiboBio, Guangzhou, China). For luciferase activity analysis, HEK293 cells were cotransfected with a combination of $200 \mathrm{ng}$ pGL3 vector containing WT or MUT 3'-UTR and $90 \mathrm{nM}$ agomir-486-5p or its negative control using Lipofectamine 2000 (Invitrogen, Thermo Fisher Scientific, Inc.). After $48 \mathrm{~h}$, the cells were harvested and luciferase activity was subjected to the Dual Luciferase Reporter Assay System (Promega, Madison, WI, USA).

\section{Statistical methods}

SPSS 19.0 software (SPSS, Inc., Chicago, IL, USA) was used for statistical analysis. Measurement data were expressed as mean \pm standard deviation (SD). Independent sample t-test was used for the intergroup comparison and one-way analysis of variance (ANOVA) was used for the comparison among multiple groups. Least significant difference (LSD) test was used for the paired comparisons $\mathrm{P}<0.05$ indicated that the difference was statistically significant.

\section{Results}

LPS inhibits cell viability and miR-486-5p expression, and induces FOXO1, inflammatory cytokines and extracellular matrix degradation in NP cells

Firstly, NP cells were treated with the indicated concentrations of LPS, and analyzed by CCK-8. We found LPS inhibited the viability of NP cells in a time and dose-dependent manner $(\mathrm{P}<0.001)$ (Fig. 1A-B). The viability of NP cells was significantly decreased by a half after $24 \mathrm{~h}$ of incubation with $100 \mathrm{ng} / \mathrm{ml}$ of LPS $(\mathrm{P}<0.001)$. Subsequently, NP cells were stimulated by $100 \mathrm{ng} / \mathrm{ml}$ of LPS for $24 \mathrm{~h}$, and then the expression levels of inflammatory cytokines, matrix degrading enzymes, and extracellular matrix (ECM)-related genes were followed by qRT-PCR and Western blot. Our findings showed that LPS significantly decreased the expression of miR-486-5p, but activated the expression of FOXO1 ( $<<0.001)$ (Fig. 1C-D). Then, LPS markedly increased the mRNA levels of inflammatory cytokines (IL-1 $\beta$, IL- 6 and TNF- $\alpha$ ) and matrix-degrading enzymes (MMP-3, MMP-13, ADAMTS-4 and ADAMTS-5), while the expressions of ECM-related genes (Aggrecan and Collagen II) were markedly down- 


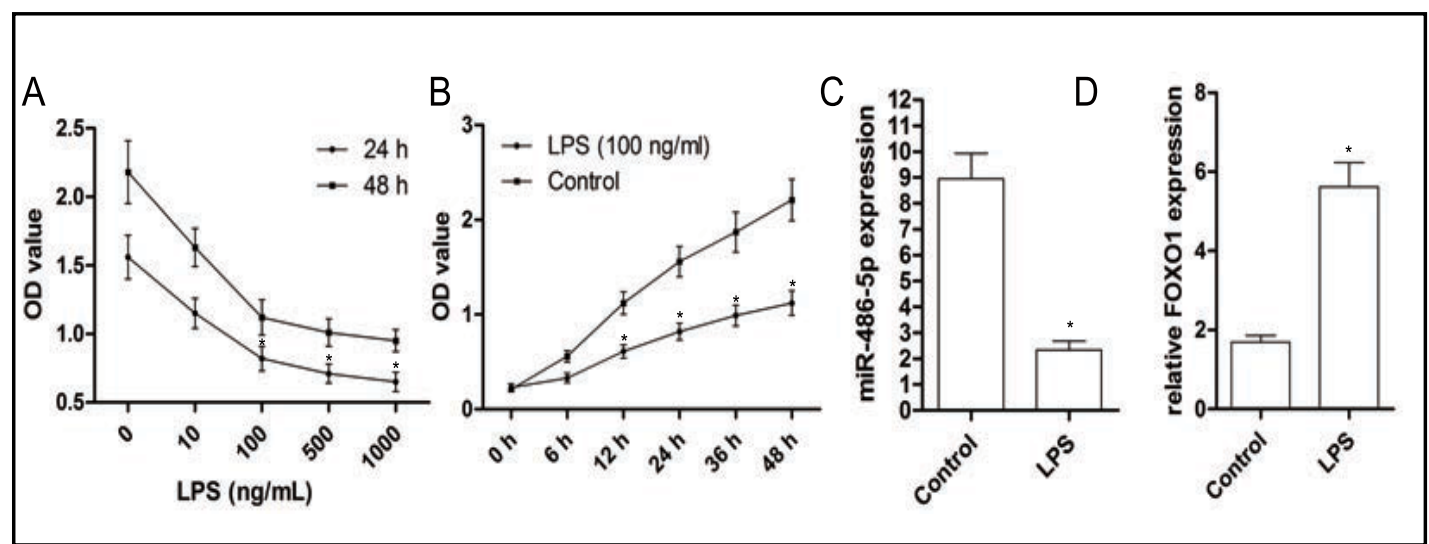

Fig. 1. LPS inhibits cell viability and miR-486-5p expression, and induces FOXO1 expression in NP cells. $(A, B)$ After LPS $(10,100,500,1000 \mathrm{ng} / \mathrm{ml})$ treatment, the viability of NP cells was measured by CCK-8 at $6,12,24,36,48 \mathrm{~h}$, respectively. Equal amounts of normal saline (LPS, $0 \mathrm{ng} / \mathrm{ml}$ ) were used as control. (C) miR-486-5p expression level was analyzed by qRT-PCR after the treatment of LPS (100 ng/ml, $24 \mathrm{~h})$. (D) The expression level of FOXO1 protein was detected by Western blot. Each experiment was assayed in triplicate independently. Values were presented as means \pm SD. $* \mathrm{P}<0.001$, v.s. control.

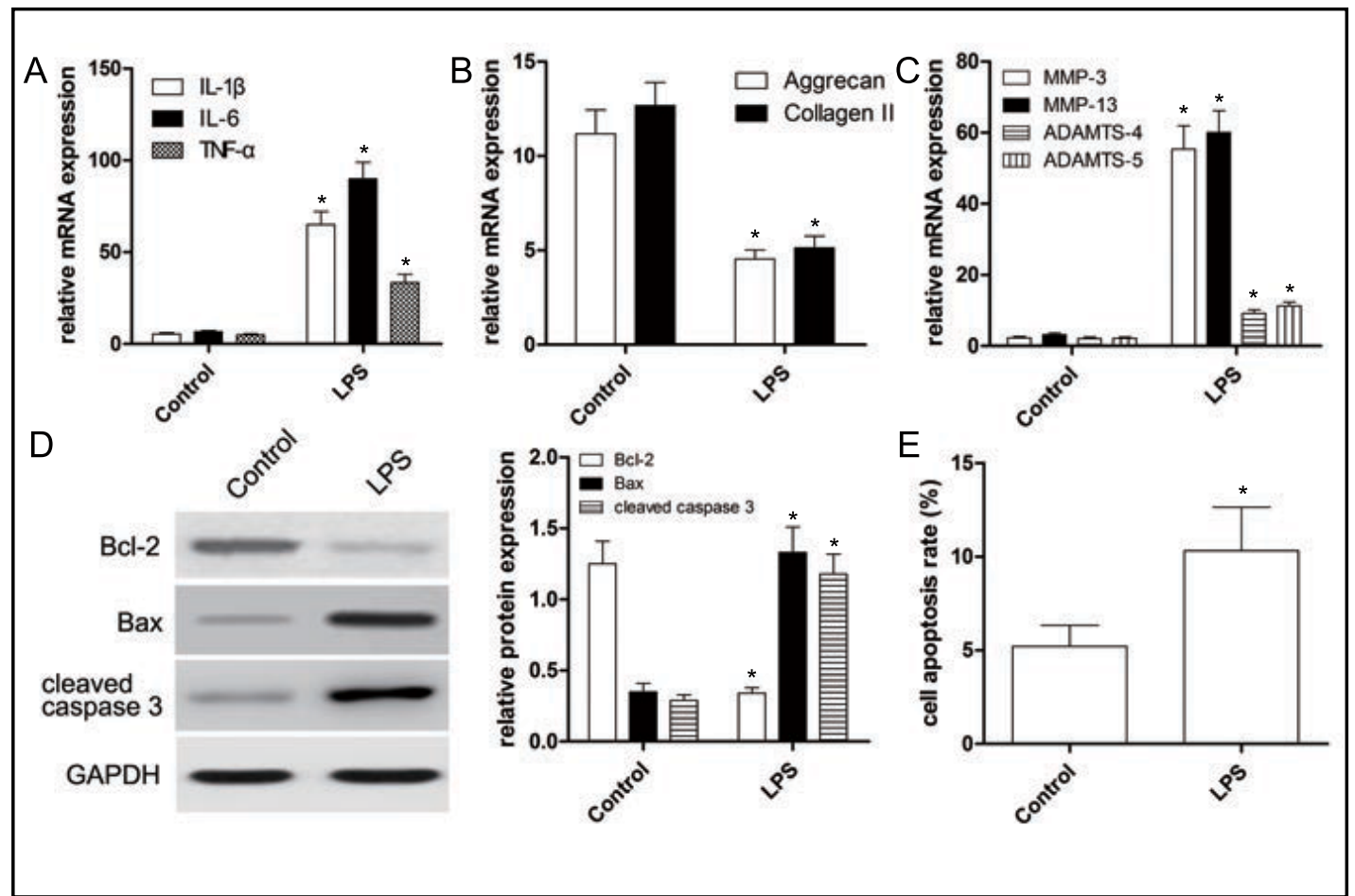

Fig. 2. LPS induces inflammatory cytokines, extracellular matrix degradation and apoptosis in NP cells. (A) qRT-PCR was used to detect the mRNA expressions of inflammatory cytokines (IL-1 $\beta$, IL- 6 and TNF- $\alpha$ ) in LPS-treated NP cells; GAPDH was used as an internal control. (B,C) qRT-PCR was used to detect the mRNA expressions of ECM genes (Aggrecan, Collagen II) and matrix degrading enzymes (MMP-3, MMP13, ADAMTS-4, and ADAMTS-5). (D) Western blot was used to detect the expressions of apoptosis-related proteins (Bcl-2, Bax and cleaved caspase 3) of NP cells. (E) Annexin/PI assay was used to measure the apoptosis rate of NP cells. Each experiment was assayed in triplicate independently. Values were presented as the means $\pm \mathrm{SD}$. $* \mathrm{P}<0.001$, v.s. control. 


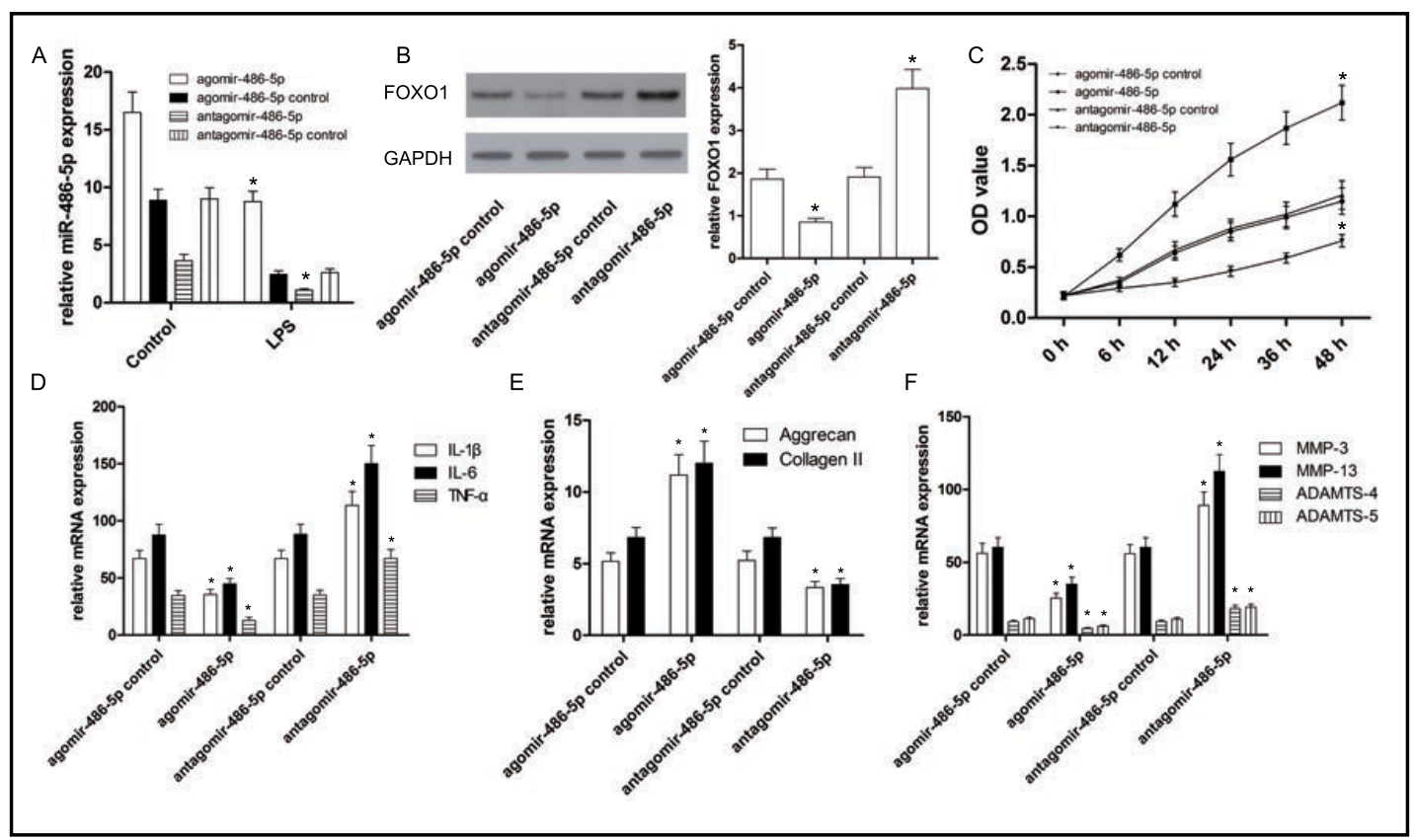

Fig. 3. The effects of miR-486-5p on LPS-induced inflammation and ECM loss of NP cells. (A) NP cells were transfected by the agomir-486-5p or antagomir-486-5p, respectively, followed by LPS stimulation. Expression of miR-486-5p was measured by qRT-PCR. (B) The bands of FOX01 protein were analyzed by Western blot, and measured by densitometry with Quantity One quantitation analysis software package. (C) The viability of NP cells was measured by CCK-8. (D) qRT-PCR validated the mRNA expressions of inflammatory cytokines in LPS-treated NP cells with agomir-486-5p or antagomir-486-5p; GAPDH was used as an internal control. (E,F) qRT-PCR validated the mRNA expressions of ECM genes and matrix degrading enzymes in LPS-treated NP cells with agomir-486-5p or antagomir-486-5p. Each experiment was assayed in triplicate independently. Data was represented as means \pm SD. $* \mathrm{P}<0.001$, v.s. control.

regulated by LPS $(\mathrm{P}<0.001)$ (Fig. 2A-C). Western blot further validated these effects above based on the protein level $(\mathrm{P}<0.001)$.

\section{LPS induces NP cell apoptosis}

To further investigate the mechanism underlying LPS-inhibited NP cell viability, we employed western blot and Annexin/PI assay to detect apoptosis of NP cells. The results showed that apoptosis-related protein cleaved caspase-3 and Bax were significantly up-regulated, but anti-apoptotic member Bcl-2 was observably down-regulated in LPSstimulated NP cells $(\mathrm{P}<0.001$, Fig. 2D). The flow-cytometric examination showed that LPS significantly increased the apoptotic proportion of NP cells by approximately two-fold compared with the untreated control $(\mathrm{P}<0.001$, Fig. 2E).

\section{miR-486-5p attenuates LPS-inhibited NP cell viability and inhibits inflammatory cytokines} in LPS-induced NP cells

To investigate the regulatory effects of miR-486-5p, we exogenously overexpressed miR486-5p in NP cells using in-vitro agomir-486-5p transfection, and endogeneously silenced miR-486-5p by transfecting antagomir-486-5p into NP cells. Then, NP cells were subjected to LPS treatment. We found miR-486-5p expression was increased by about3.1-fold accompanied by decreased FOX01 expression (Fig. 3A-B). However, antagomir-486-5p obviously reduced the level of miR-486-5p by approximately $60 \%$, and obviously improved the viability of NP cells (Fig. 3A, 3C). On the other hand, miR-486-5p over-expression obviously inhibited LPSincreased mRNA levels of IL-1 $\beta$, IL- 6 and TNF- $\alpha$ compared with controls $(\mathrm{P}<0.001$, Fig. 3D), while miR-486-5p inhibition significantly enhanced LPS-increased expressions of IL-1 $\beta$, IL-6 


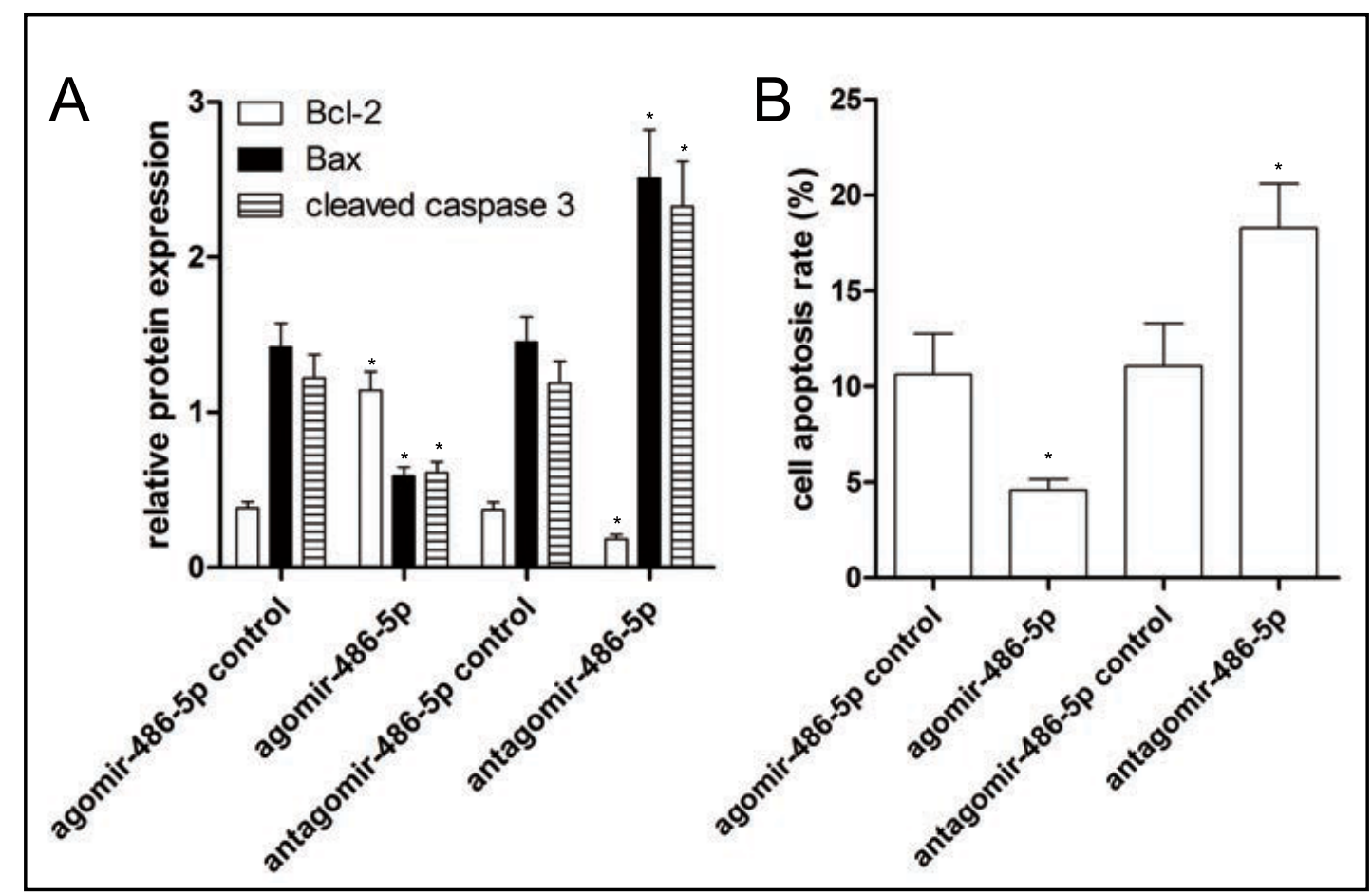

Fig. 4. The effects of miR-486-5p on LPS-induced apoptosis of NP cells. (A) Western blot was used to detect the expressions of apoptosis-related proteins. (B) The apoptosis rate of NP cells in each group was detected by flow cytometry. Each experiment was assayed in triplicate independently. Data was represented as means \pm SD. ${ }^{*} \mathrm{P}<0.001$, v.s. control.

and TNF- $\alpha$ mRNAs in contrast with those in the antagomir-486-5p control group $(\mathrm{P}<0.001$, Fig. 3D). Western blot further identified these effects $(\mathrm{P}<0.001)$.

miR-486-5p promotes the expression of ECM genes and inhibits synthesis of ECM-degrading enzymes in LPS-induced NP cells

To further affirm the regulatory effects of miR-486-5p on the expression of ECM genes and ECM-degrading enzymes in NP cells, NP cells were transfected with agomir-486$5 p$, antagomir-486-5p, or their respective controls. Then NP cells were subjected to LPS stimulation. We found that levels of Aggrecan and Collagen II in agomir-486-5p group were obviously higher than those in the agomir-486-5p control, while the levels of Aggrecan and Collagen II in the antagomir-486-5p group showed a significant decrease than those in the antagomir-486-5p control $(\mathrm{P}<0.001$, Fig. $3 \mathrm{E})$. Moreover, over-expression of miR-486-5p inhibited LPS-increased expressions of matrix degrading enzymes than control $(\mathrm{P}<0.001$, Fig. 3F). However, miR-486-5p inhibition remarkably promoted the expressions of matrix degrading enzymes in NP cells stimulated by LPS, leading to the higher levels of matrix degrading enzymes than LPS treatment alone $(\mathrm{P}<0.001$, Fig. $3 \mathrm{~F})$.

\section{miR-486-5p protects NP cells against LPS-induced apoptosis}

We found that the protein expressions of Bax and cleaved caspase-3 were markedly reduced by agomir-486-5p, whereas Bcl-2 was obviously elevated by agomir-486-5p in NP cells as compared with that in the agomir-486-5p control group $(\mathrm{P}<0.001$, Fig. 4A). NP cells with antagomir-486-5p exhibited the opposite results. In addition, in comparison with the agomir-486-5p control group, the apoptosis rate of NP cells dramatically decreased following transfection with the agomir-486-5p ( $\mathrm{P}<0.001$, Fig. 4B). However, antagomir-486$5 p$ obviously exacerbated the apoptosis of NP cells compared with the antagomir-486-5p control group $(\mathrm{P}<0.001$, Fig. 4B). 


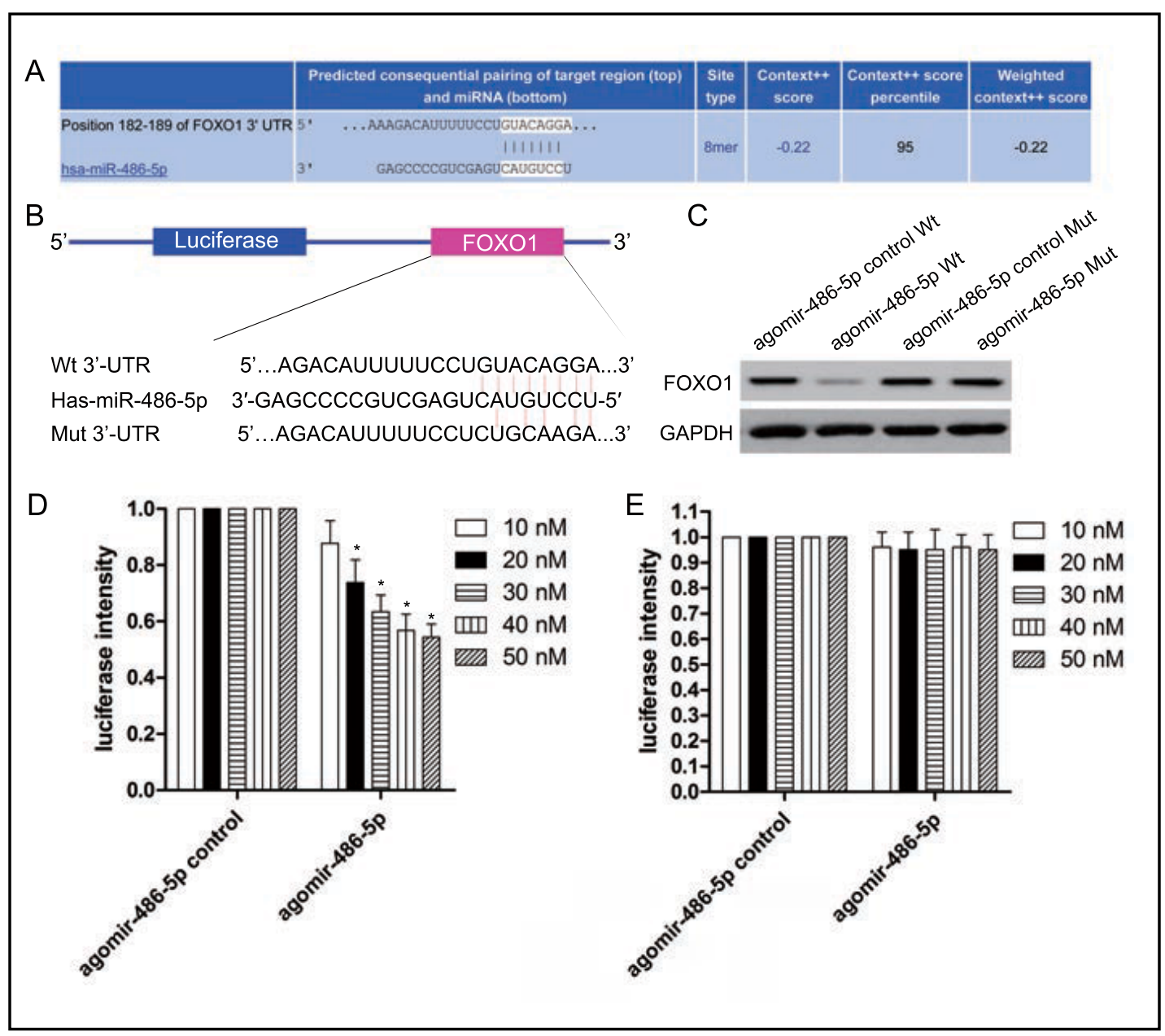

Fig. 5. FOXO1 is a direct target of miR-486-5p. (A) The prediction of the binding between miR-486-5p and FOX01 was conducted by TargetScan. (B) Seed sequences of miR-486-5p in the Wt and Mut 3'-UTR of F0X01. (C) The expression of FOX01 was detected by Western blot in HEK293 cells after co-transfection of agomir-486-5p and/or 3'-UTR (Wt or Mut) of FOXO1. (D,E) Luciferase reporter assay was performed to verify the binding of miR-486-5p in the Wt or Mut 3'-UTR of FOX01. Each experiment was assayed in triplicate independently. Data was represented as means \pm SD. $* \mathrm{P}<0.001$, v.s. control.

\section{FOXO1 is a direct target of miR-486-5p}

We found that the 3 '-UTR of FOXO1 possesses sequences complementary to the miR-486$5 p$ seed sequences using TargetScan algorithms (Fig. 5A). Next, luciferase reporter vectors containing wild-type (Wt) or mutated (Mut) FOXO1 3'-UTR sequences were generated and transfected into HEK293 cells (Fig. 5B). Western blot analysis revealed the decreased expression of FOXO1 protein in HEK293 cells co-transfected with Wt FOXO1 3'-UTR and agomir-486-5p $(\mathrm{P}<0.001$, Fig. 5C). The results revealed that over-expression of miR-486$5 p$ effectively reduced luciferase activity of the wild type reporter genes, instead of mutant reporter genes (Fig. 5D-E), indicating that miR-486-5p directly targets the 3'-UTR of FOXO1.

FOXO1 is involved in the regulatory effects of miR-486-5p in NP cells

Here, we explored whether FOXO1 was involved in miR-486-5p-induced biological effects in NP cells. The pcDNA-FOXO1 or pcDNA-control was transfected into miR-486$5 p$-overexpressing NP cells. The results showed that over-expression of FOXO1 decreased agomir-486-5p-improved NP cell viability. Moreover, over-expression of FOXO1 antagonized the effect of agomir-486-5p on the mRNA expressions of inflammation cytokines $(\mathrm{P}<0.001$, 


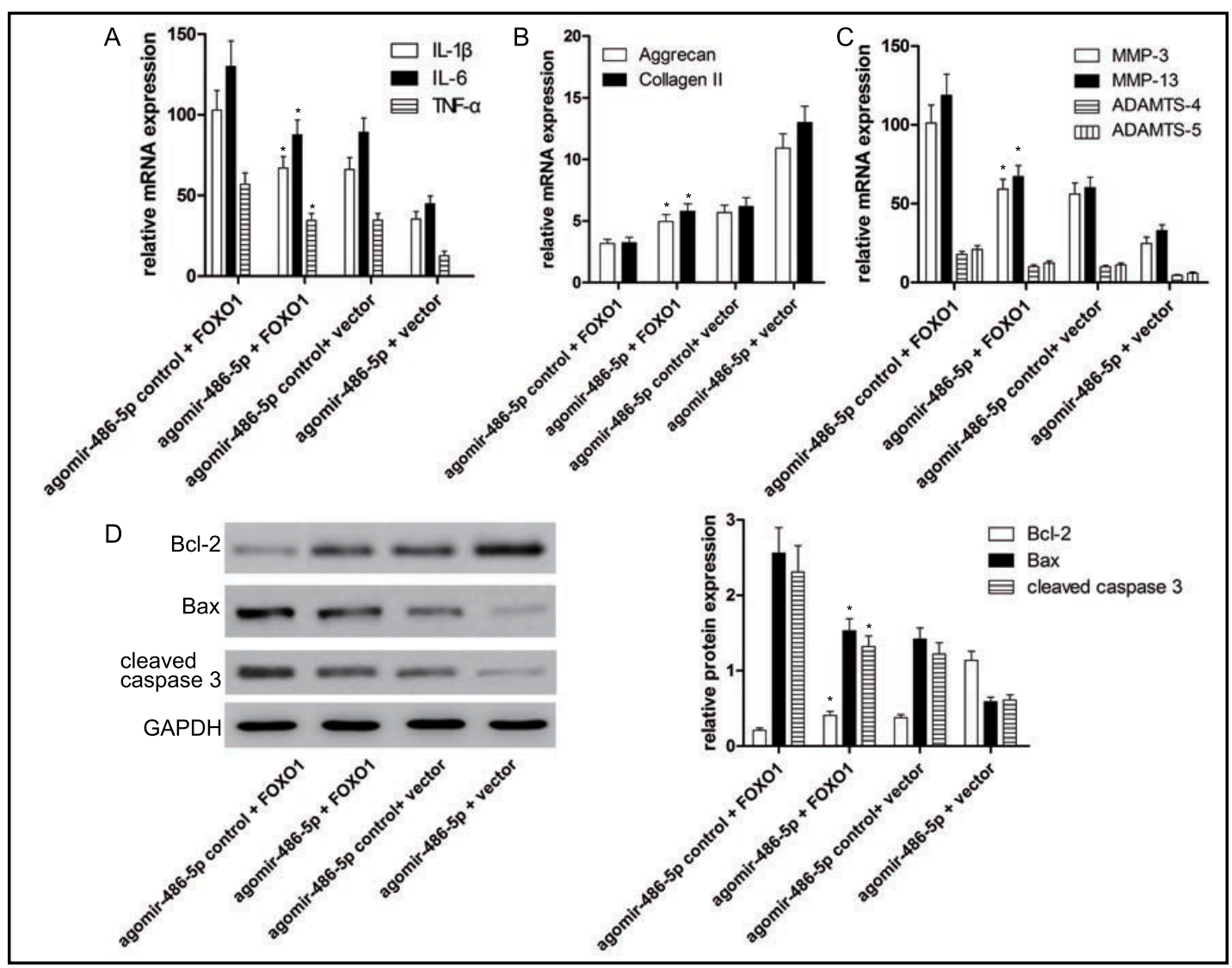

Fig. 6. FOXO1 is involved in the regulatory effects of miR-486-5p in NP cells. (A) qRT-PCR was performed for the mRNA expressions of inflammatory cytokines. (B,C) qRT-PCR was performed for the mRNA expressions of ECM genes and matrix degrading enzymes. (D) Western blot was used to detect the expressions of apoptosis-related proteins. Bands were measured by densitometry with Quantity One quantitation analysis software package. Each experiment was assayed in triplicate independently. Data was represented as means \pm SD. ${ }^{*} \mathrm{P}<0.001$, v.s. control.

Fig. 6A). Also, over-expression of FOX01 inhibited miR-486-5p-recovered Aggrecan and Collagen II mRNA expressions, while FOX01 promoted the mRNA expressions of miR-486$5 p$-inhibited matrix degrading enzymes $(\mathrm{P}<0.001$, Fig. 6B-C). Western blot further identified these effects $(\mathrm{P}<0.001)$.

In addition, the expression of Bax and cleaved caspase 3 were about two-fold higher in NP cells with FOXO1 over-expression, while miR-486-5p partially reversed these effects $(\mathrm{P}<0.001$, Fig. 6D). Bcl-2 expression was descended to $48.9 \%$ in NP cells with FOXO1 overexpression, and partially improved by miR-486-5p ( $\mathrm{P}<0.001$, Fig. 6D). At the same time, Annexin/PI assay showed FOX01 over-expression aggravated LPS-induced apoptosis, and affected miR-486-5p-protected cell viability $(\mathrm{P}<0.01)$.

\section{Discussion}

It is well established that cell apoptosis, inflammatory response and ECM degradation contribute to the development of IDD [14-16]. Some miRNAs that regulate these processes have been linked to IDD $[13,17]$. Therefore, identification of IDD-associated miRNAs and elucidation of their roles in IDD may be important for developing novel targets for IDD therapy. In this study, we demonstrated that LPS inhibited miR-486-5p expression, and induced inflammatory cytokines and apoptosis in NP cells. In-vitro assays revealed that miR- 


\section{Cellular Physiology Cell Physiol Biochem 2019;52:109-118 \\ \begin{tabular}{ll|l} 
and Biochemistry & $\begin{array}{l}\text { DOl: 10.33594/000000008 } \\
\text { Published online: } 18 \text { February } 2019\end{array}$ & $\begin{array}{l}\text { O } 2019 \text { The Author(s). Published by } \\
\text { Cell Physiol Biochem Press GmbH\&Co. KG }\end{array}$ \\
\cline { 2 - 3 }
\end{tabular} \\ Chai et al.: miR-486-5p Regulates FOXO1 in IDD}

486-5p over-expression inhibited LPS-induced the expressions of inflammatory cytokines, and prevented cell apoptosis, indicating that miR-486-5p regulated inflammatory processes, and played a protective role in NP cells. As reported, some miRNAs also regulate matrix degradation and synthesis of ECM-degrading enzymes in NP cells. Liu et al. reported that miR-7 regulated IL-1 $\beta$-induced ECM degeneration by targeting GDF5 in human nucleus pulposus cells [18]. In our work, LPS repressed the expressions of ECM genes and induced ECM-degrading enzymes. However, miR-486-5p antagonized LPS-induced ECM injury effects. These findings suggested miR-486-5p indeed participates in the development of IDD.

According to recent reports, the expressions of inflammatory cytokines and ECMdegrading enzymes were regulated by FOXO1 $[19,20]$. Under basal conditions, activated FOX01 is translocated into the nucleus in response to different types of stresses [21, 22]. Further, FOX01 targets the promoter of related cytokine gene locus to directly regulate the expressions of inflammatory cytokines [23]. Because Foxo transcription factors contain both DBD and protein interaction domain, they exert some effects in a DNA dependent or independent fashion. FOXO1 plays a vital role in the cell apoptosis, cell cycle, DNA damage repair, oxidative stress, and basic metabolisms. However, the detailed mechanisms underlying FOX01-mediated development of IDD remain unknown. Here, we found FOXO1 activation by LPS or over-expression indeed promoted the expressions of inflammatory cytokines and extracellular matrix degradation in NP cells, which made us assume that FOXO1 may be a target of miR-486-5p.

Since miRNAs exert their biological effects through binding to the 3'-UTR of target mRNAs to negatively modulate the expressions of their target genes, including inhibition of translation or degradation of specific mRNAs. Here, we applied putative bioinformatics prediction databases, and predicted FOXO1 is a potential target of miR-486-5p. Previous studies reported FOXO1 can be targeted by many kinds of miRNAs. Jia X et al. demonstrated that miR-16 affected myoblast proliferation and apoptosis through directly repressing FOXO1 activities [24]. He W et al. suggested that miR-374a promoted the proliferation capacity of human osteosarcoma via down-regulating FOXO1 expression [25]. Mao XP also found that miR-135a enhanced bladder cancer cell proliferation through regulating FOXO1 at a posttranscriptional level [26]. Consistently, our luciferase reporter assay revealed that FOXO1 is indeed a direct target of miR-486-5p, by which miR-486-5p exerted its functions in NP cells.

\section{Conclusion}

In conclusion, miR-486-5p expression was down-regulated in LPS-induced NP cells. miR-486-5p improved NP cell viability, and inhibited inflammation cytokines and ECM degradation partly via inhibition of FOXO1 expression, which may contribute to the biological therapy of IDD.

\section{Disclosure Statement}

The authors have declared that no conflicts of interest exists.

\section{References}

1 Andersson GB: Epidemiological features of chronic low-back pain. Lancet 1999;354:581-585.

- 2 Waddell G: Low back pain: a twentieth century health care enigma. Spine 1996;21:2820-2825.

- 3 Guehring T, Omlor GW, Lorenz H, Bertram H, Steck E, Richter W, Carstens C, Kroeber M: Stimulation of gene expression and loss of anular architecture caused by experimental disc degeneration-an in vivo animal study. Spine 2005;30:2510-2515. 


\section{Cellular Physiology Cell Physiol Biochem 2019;52:109-118 \begin{tabular}{ll|l|l|l}
\hline DOI: 10.33594/000000008 2019 The Author(s). Published by & C
\end{tabular} and Biochemistry Published online: 18 February 2019 Cell Physiol Biochem Press GmbH\&Co. KG \\ Chai et al.: miR-486-5p Regulates FOXO1 in IDD}

- 4 Wang B, Wang D, Yan T, Yuan H: miR-138-5p promotes TNF- $\alpha$-induced apoptosis in human intervertebral disc degeneration by targeting SIRT1 through PTEN/PI3K/Akt signaling. Exp Cell Res 2016;345:199-205.

- 5 Stokes IA, Iatridis JC: Mechanical conditions that accelerate intervertebral disc degeneration: overload versus immobilization. Spine 2004;29:2724-2732.

6 Zhang WL, Chen YF, Meng HZ, Du JJ, Luan GN, Wang HQ, Yang MW, Luo ZJ: Role of miR-155 in the regulation of MMP-16 expression in intervertebral disc degeneration. J Orthop Res 2017;35:1323-1334.

7 Guo H, Ingolia NT, Weissman JS, Bartel DP: Mammalian microRNAs predominantly act to decrease target mRNA levels. Nature 2010;466:835-840.

8 Hwang HW, Mendell JT: MicroRNAs in cell proliferation, cell death, and tumorigenesis. Br J Cancer 2007;96:R40-44.

9 Teague EM, Print CG, Hull ML: The role of microRNAs in endometriosis and associated reproductive conditions. Hum Reprod Update 2010;16:142-165.

10 Smyth LA, Boardman DA, Tung SL, Lechler R, Lombardi G: microRNAs affect dendritic cell function and phenotype. Immunology 2015;144:197-205.

- 11 Liu G, Cao P, Chen H, Yuan W, Wang J, Tang X: miR-27a regulates apoptosis in nucleus pulposus cells by targeting PI3K. PLoS One 2013;8:e75251.

- 12 Liu H, Huang X, Liu X, Xiao S, Zhang Y, Xiang T, Shen X, Wang G, Sheng B: miR-21 promotes human nucleus pulposus cell proliferation through PTEN/AKT signaling. Int J Mol Sci 2014;15:4007-4018.

- 13 Ji ML, Zhang XJ, Shi PL, Lu J, Wang SZ, Chang Q Chen H, Wang C: Downregulation of microRNA-193a-3p is involved in invertebral disc degeneration by targeting MMP14. J Mol Med (Berl) 2016;94:457-468.

14 Ye D, Dai L, Yao Y, Qin S, Xie H, Wang W, Liang W: miR-155 Inhibits Nucleus Pulposus Cells' Degeneration through Targeting ERK 1/2. Dis Markers 2016;2016:6984270.

- 15 Gruber HE, Norton HJ, Sun Y, Hanley EN Jr: Crystal deposits in the human intervertebral disc: Implications for disc degeneration. Spine J 2007;7:444-450.

16 Shamji MF, Setton LA, Jarvis W, So S, Chen J, Jing L, Bullock R, Isaacs RE, Brown C, Richardson WJ: Proinflammatory cytokine expression profile in degenerated and herniated human intervertebral disc tissues. Arthritis Rheum 2010;62:1974-1982.

17 Wang H, Hao P, Zhang H, Xu C, Zhao J: MicroRNA-223 inhibits lipopolysaccharide-induced inflammatory response by directly targeting Irak1 in the nucleus pulposus cells of intervertebral disc. IUBMB Life 2018;70:479-490.

- 18 Liu W, Zhang Y, Xia P, Li S, Feng X, Gao Y, Wang K, Song Y, Duan Z, Yang S, Shao Z, Yang C: MicroRNA-7 regulates IL-1beta-induced extracellular matrix degeneration by targeting GDF5 in human nucleus pulposus cells. Biomed Pharmacother 2016;83:1414-1421.

19 Li Z, He Q, Zhai X, You Y, Li L, Hou Y, He F, Zhao Y, Zhao J: Foxo1-mediated inflammatory response after cerebral hemorrhage in rats. Neurosci Lett 2016;629:131-136.

- 20 Liu Z, Zhou K, Fu W, Zhang H: Insulin-Like Growth Factor 1 Activates PI3k/Akt Signaling to Antagonize Lumbar Disc Degeneration. Cell Physiol Biochem 2015;37:225-232.

- 21 Maekawa T, Maniwa Y, Doi T, Nishio W, Yoshimura M, Ohbayashi C, Hayashi Y, Okita Y: Expression and localization of FOXO1 in non-small cell lung cancer. Oncol Rep 2009;22:57-64.

- 22 Malik S, Awasthi A: Transcriptional Control of Th9 Cells: Role of Foxo1 in Interleukin-9 Induction. Front Immunol 2018;9:995.

- 23 Essaghir A, Dif N, Marbehant CY, Coffer PJ, Demoulin JB: The transcription of FOXO genes is stimulated by FOXO3 and repressed by growth factors. J Biol Chem 2009;284:10334-10342.

24 Jia X, Ouyang H, Abdalla BA, Xu H, Nie Q Zhang X: miR-16 controls myoblast proliferation and apoptosis through directly suppressing Bcl2 and FOXO1 activities. Biochim Biophys Acta Gene Regul Mech 2017;1860:674-684.

- 25 He W, Feng L, Xia D, Han N: miR-374a promotes the proliferation of human osteosarcoma by downregulating FOX01 expression. Int J Clin Exp Med 2015;8:3482-3489.

- 26 Mao XP, Zhang LS, Huang B, Zhou SY, Liao J, Chen LW, Qiu SP, Chen JX: miR-135a enhances cellular proliferation through post-transcriptionally regulating PHLPP2 and FOXO1 in human bladder cancer. J Transl Med 2015;13:86. 